\title{
Salvage cytoreductive surgery for pleural dissemination of low-grade endometrial stromal sarcoma using rib-cross thoracotomy with argon beam coagulation: strategies to achieve a grossly complete resection - a case report
}

\author{
Noriaki Sakakura ${ }^{1}$, Masahiko Mori $^{2}$, Satoshi Tsukushi ${ }^{3}$, Takeo Nakada ${ }^{1}$, Yusuke Takahashi ${ }^{1}$, \\ Hiroaki Kuroda ${ }^{1}$ \\ ${ }^{1}$ Department of Thoracic Surgery, Aichi Cancer Center Hospital, Nagoya, Japan; ${ }^{2}$ Department of Gynecologic Oncology, Aichi Cancer Center \\ Hospital, Nagoya, Japan; ${ }^{3}$ Department of Orthopaedic Surgery, Aichi Cancer Center Hospital, Nagoya, Japan \\ Correspondence to: Noriaki Sakakura, MD, PhD. Department of Thoracic Surgery, Aichi Cancer Center Hospital, 1-1 Kanokoden, Chikusa-ku, \\ Nagoya 464-8681, Japan. Email: nsakakura@aichi-cc.jp.
}

\begin{abstract}
We present a case of low-grade endometrial stromal sarcoma (LG-ESS) treated with salvage resection of disseminated nodules in the unilateral thorax by using the rib-cross thoracotomy approach with argon beam coagulation (ABC). To our knowledge, this is the first reported case of intrathoracic pleural dissemination of LG-ESS for which grossly complete resection was achieved. A 69-year-old woman was found to have multiple tumors of various sizes and locations in the right thoracic cavity, including a 10 -cmsized tumor invading the right ventral thoracic wall and diaphragm. At age 50 years, the patient underwent a hysterectomy for uterine LG-ESS. The thoracic lesions were diagnosed as disseminated recurrence of the LG-ESS. As the lesions were enlarged despite hormone therapy, including medroxyprogesterone acetate and letrozole, in addition to platinum-based chemotherapy, salvage tumor debulking was considered. Ribcross thoracotomy was adopted; the sixth, seventh, and eighth ribs were cut along the mid-axillary line, and the thorax was entered posteriorly at the fifth intercostal space and anteriorly at the eighth intercostal space, providing wide exposure of the caudal side of the thorax. The largest lesion was excised with combined resection of the soft tissue of the chest wall and diaphragm. Twelve gross nodules were resected, and smaller lesions were cauterized using ABC. Combined with magnifying thoracoscopy and ABC, a grossly visible complete resection of the disseminated tumors could be achieved. Although 4 years after the surgery, some small nodules were observed in the ipsilateral thorax, the patient remains in good health at age 73 years, 23 years after the surgery for the primary uterine LG-ESS, while continually receiving letrozole therapy. For low-grade malignant tumors with no other effective treatment available, if technically possible, cytoreductive surgery can be chosen in a salvage setting for disease control, even with dissemination. In this report accompanied with a short video, we discuss the surgical treatment used, including an uncommon type of modified extended thoracotomy and $\mathrm{ABC}$, to achieve a grossly complete resection of pleural dissemination of slowly progressing soft tissue malignant tumors.
\end{abstract}

Keywords: Low-grade endometrial stromal sarcoma (LG-ESS); rib-cross thoracotomy; argon beam coagulation (ABC); salvage cytoreductive surgery; case report

Received: 16 March 2021; Accepted: 09 April 2021; Published: 30 June 2021.

doi: 10.21037/asj-21-12

View this article at: http://dx.doi.org/10.21037/asj-21-12 


\section{Introduction}

Low-grade endometrial stromal sarcoma (LG-ESS) is a rare type of primary uterine malignancy (1), which is unfamiliar to thoracic surgeons. Although it is described as low-grade on the basis of a 5 -year overall survival rate of approximately $91 \%$ from the initial treatment of primary lesions (2), distant metastasis to the thorax can occur over the long term (3-7), and thoracic surgeons may be consulted. In this report, which is accompanied with a short video, we present a case of LG-ESS treated with salvage tumor debulking surgery using the rib-cross thoracotomy approach (8-10) combined with argon beam coagulation $(\mathrm{ABC})$ for disseminated nodules in the unilateral thorax. To our knowledge, this is the first reported case of resection of intrathoracic pleural dissemination of LG-ESS. We present the following case in accordance with CARE reporting checklist (available at https://asj.amegroups.com/article/ view/10.21037/asj-21-12/rc).

\section{Case presentation}

A 69-year-old female patient who was a non-smoker complained of discomfort in her anterior chest at the age of 68 years. Computed tomography (CT) revealed multiple lesions of various sizes in the right thoracic cavity, including a $10-\mathrm{cm}$-sized tumor invading the right ventral thoracic wall and diaphragm (Figure 1A). On positron emission tomography, the lesion showed an accumulation of $18 \mathrm{~F}$-fluorodeoxyglucose with a maximum standardized uptake value of 4.0 (Figure 1B). At another hospital, she underwent a left mastectomy for breast cancer at age 49 years and a total hysterectomy and bilateral salpingooophorectomy for the treatment of uterine neoplasm at age 50 years. The uterine tumor was a LG-ESS, and complete resection was achieved. The 5 -year postoperative followup was completed without any recurrence. The tumors in the chest were initially diagnosed as lung cancer or a certain type of sarcoma on the basis of CT-guided needle biopsy findings; therefore, treatment with three courses of platinum doublet chemotherapy with carboplatin and etoposide was initiated. However, the tumors grew, and the patient was thus referred to our hospital. After review of the biopsy specimen, a diagnosis of pleural dissemination of the LG-ESS was finally made. First, $600-\mathrm{mg} /$ day medroxyprogesterone acetate (MPA) was administered for 3 months, which resulted in tumor growth. Next, $2.5-\mathrm{mg} /$ day letrozole, an aromatase inhibitor, was administered for 4 months; however, the lesions became more enlarged. The multidisciplinary team at the sarcoma center of our institution, which consisted of gynecologists, surgeons and medical oncologists specializing in soft tissue sarcoma, and thoracic surgeons, discussed whether salvage tumor debulking surgery would be suitable for this case. A consensus was reached that such surgery for the disseminated nodules, with concomitant hormone therapy, might be of some value, especially for the large lesions.

For combined resection of adjacent organs, including the thoracic wall and diaphragm, and the resection and ablation of the multiple disseminated lesions that had grown widely in the thoracic cavity, we adopted rib-cross thoracotomy (8-10), which is an extended modification of posterolateral thoracotomy. The patient was placed in the conventional left lateral decubitus position and managed with general anesthesia and double-lumen intubation. An approximately 30-cm S-shaped skin incision was made, and the latissimus dorsi muscle was cut, and the serratus anterior muscle was split. The sixth, seventh, and eighth ribs, and the intercostal muscles and vessels were cut along the mid-axillary line, and the thorax was entered posteriorly at the fifth intercostal space and anteriorly at the eighth intercostal space (Figures $2 A, B)$ to expose the entire caudal side of the thoracic cavity. Lesions of various sizes were revealed (Figure 2C); the largest lesion was excised with combined resection of the soft tissue of the thoracic wall around the fifth and sixth ribs and the diaphragm. Twelve grossly visible nodules were resected, some of which were resected using partial lung resection. Moreover, the clusters of smaller disseminated lesions of a few millimeters were cauterized using $\mathrm{ABC}$ (System 7550, Electrosurgical Generator with ABC Models, ConMed Corporation, Utica, NY, USA) at $100 \mathrm{~W}$ (Figure $2 D)$. Finally, almost the entire thorax was widely exposed using two thoracic retractors (Figure 2E). Each tumor bed and its surrounding region and small disseminated nodules of the lung surface were also cauterized. Under magnifying thoracoscopy, recognizable disseminated nodules were cauterized as much as possible; a grossly visible complete resection was achieved. The chest wall was fixed using rib pins (Super Fixsorb 30; Takiron Co., Ltd., Osaka, Japan) and No. 5 Ethibond nonabsorbable braided polyester sutures (Ethicon, Inc., Somerville, NJ, USA), and closed (Figure 2F). Video 1 demonstrates these surgical findings and procedures. The surgery took $5 \mathrm{~h} 45 \mathrm{~min}$, and the blood loss volume was $430 \mathrm{~g}$. For postoperative analgesia, loxoprofen sodium (180 mg/day) and oral oxycodone $(20 \mathrm{mg} /$ day $)$ were regularly administered for approximately 

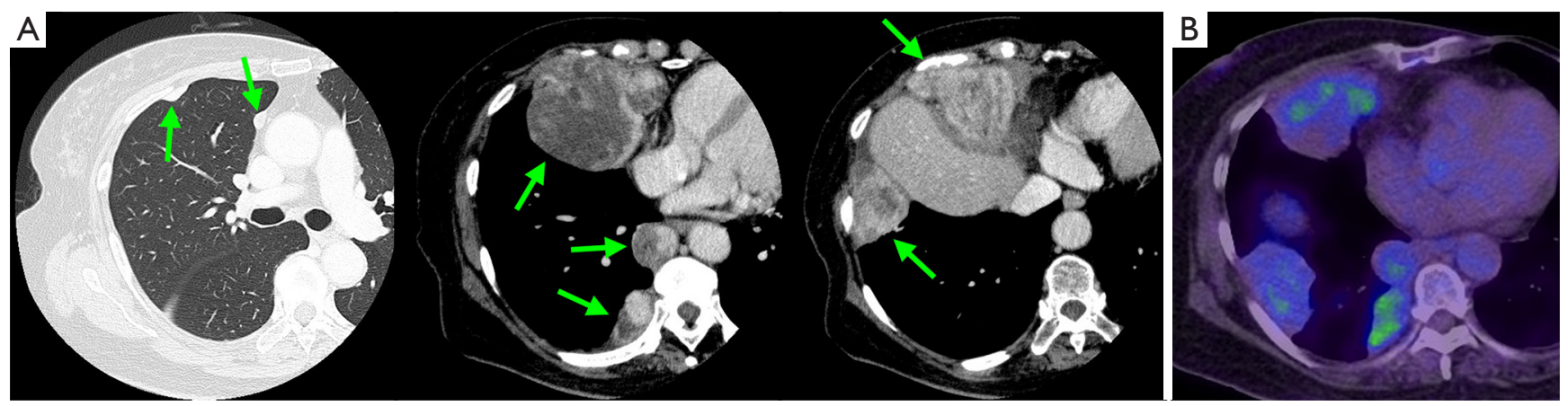

Figure 1 Radiological findings. (A) The computed tomography image shows disseminated nodules of various sizes and locations (arrows). (B) The positron emission tomography image shows multiple lesions exhibiting a relatively low uptake.

A

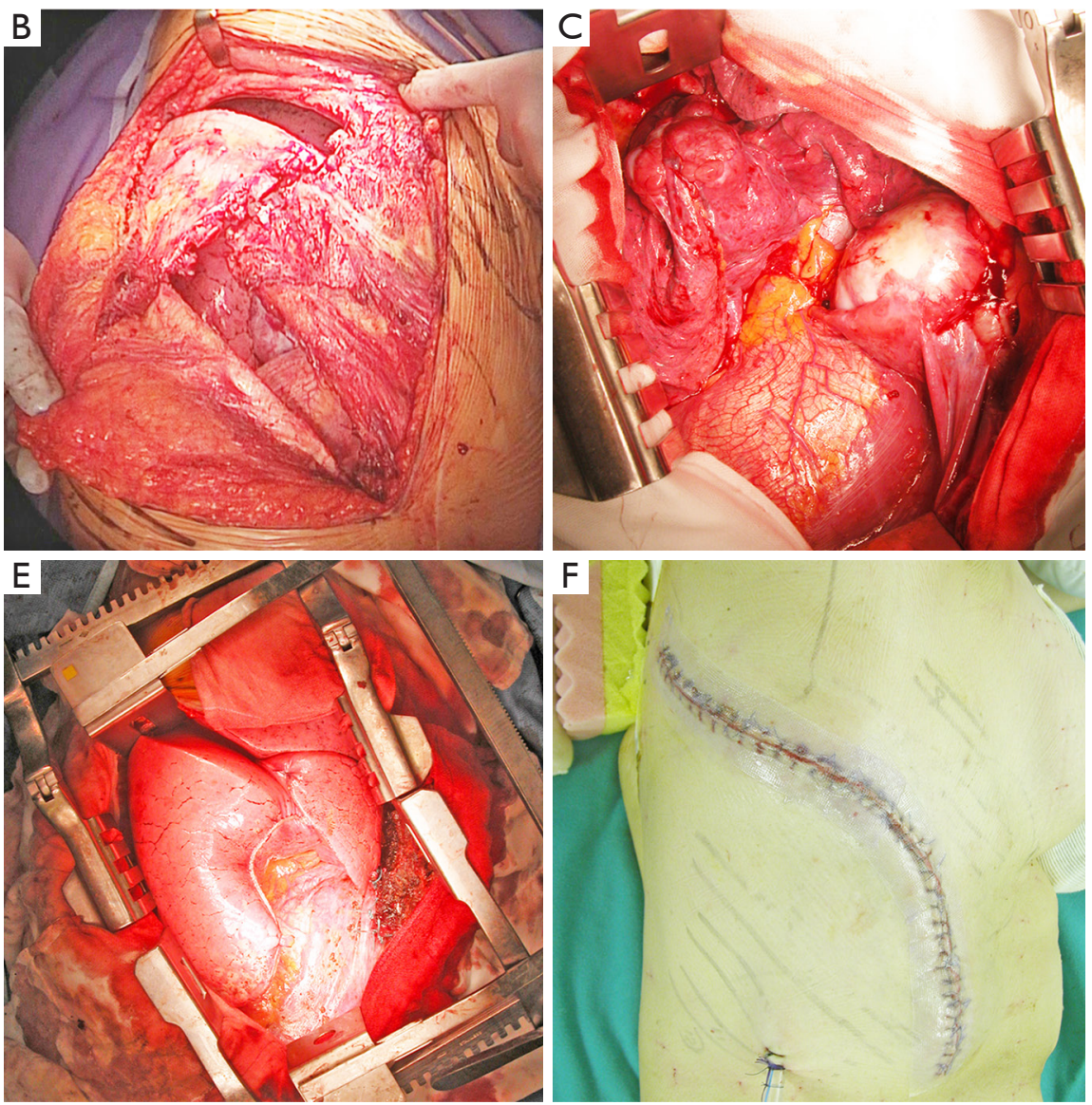

Figure 2 Surgery. (A,B) The rib-cross thoracotomy. To open the thorax, retractors are used posteriorly at the cranial intercostal space and anteriorly at the caudal intercostal space (arrows). The thoracotomy location and length can be altered along the axillary line depending upon the lesion size and location of the intended thoracic cavity. (C) Intrathoracic findings. Tumors of various sizes can be observed. (D) Cauterization of the disseminated nodules using argon beam coagulation. (E) The surgical view. The whole thoracic cavity is widely exposed. (F) The incision line. 




Video 1 Surgical procedures and intraoperative findings.

1 month. The postoperative course was uneventful.

After the operation, oral administration of $2.5-\mathrm{mg}$ /day letrozole was continued. Two years after the surgery, small nodules were detected around the diaphragm on a routine CT. Four years after the surgery, some disseminated nodules of 1 to $2 \mathrm{~cm}$ in size were observed in the right thoracic cavity. The patient remains in good health at age 73 years, 23 years after the surgery for the primary uterine LG-ESS, while continually receiving letrozole therapy. As a future treatment option, single-agent chemotherapy with doxorubicin or a molecular target therapy with pazopanib, or new antitumor agents, including trabectedin and eribulin, can be considered, although their efficacies may be limited. All procedures performed in this study were in accordance with the ethical standards of the institutional and national research committees and with the Helsinki Declaration (as revised in 2013). Written informed consent was obtained from the patient for publication of this manuscript and any accompanying images. A copy of the written consent is available for review by the editorial office of this journal.

\section{Discussion}

Uterine sarcomas comprise a heterogeneous group of rare tumors of the uterine musculature and connective tissue, and are classified as follows (1): leiomyosarcoma, LG-ESS, high-grade (HG) ESS, undifferentiated uterine sarcoma, adenosarcoma, rhabdomyosarcoma, and malignant-type perivascular epithelioid cell tumor. ESSs represent only approximately $0.2 \%$ of all uterine malignancies but account for approximately $7 \%$ of all uterine sarcomas (1). ESSs are considered the second most frequent type of uterine mesenchymal neoplasia after uterine leiomyosarcoma. LG-ESS is often detected in the early fifth decade of life on average and often recognized owing to abnormal bleeding, abdominal pain, or pelvic masses, but may often be also asymptomatic. Metastatic lesions may provide an opportunity for detection. The possible differential diagnoses of LGESS are endometrial stromal nodule, cellular leiomyoma, adenomyosis, adenosarcoma, adenomyoma, and intravascular leiomyomatosis. Reportedly, the median follow-up time of patients with LG-ESS was 75 months, and the 5 -year overall survival rate was approximately $91 \%$ in patients with LG-ESS and 33\% in those with HG-ESS (2). LG-ESS is characterized by late recurrences even in patients with stage I disease, which suggests the need for long-term follow-up. Histopathologically, LGESS is characterized by uniformly bland cells resembling endometrial stromal cells. Immunohistochemically, the tumor is usually positive for CD10, and progesterone and estrogen receptors. Moreover, LG-ESS is classified into 3 categories based on molecular genetic abnormalities as follows: translocation $\mathrm{t}(7 ; 17)(\mathrm{p} 15 ; \mathrm{q} 21) ; 6 \mathrm{p} 21-$ rearrangements; and X;22 or 7-rearrangements (11). Cell lines for genetic and molecular biological investigations have been developed (12). As LG-ESS is basically hormone sensitive, hormone therapy with MPA or letrozole, in addition to chemotherapy, can contribute to the improvement of prognosis in patients with residual disease, progressive disease, or recurrence (3-7). Although few cases of thoracic metastases of LG-ESS other than those to the lung have been reported, distant metastatic lesions of recurrent LG-ESS can be repeatedly resected because of the slow progression of the disease, possibly resulting in better prognosis (7).

Rib-cross thoracotomy was reported by Tsubota in 1996 as an extended modification of posterolateral thoracotomy for approaching large thoracic lesions (8). Initially, the sixth rib and intercostal muscles and vessels are cut along the mid-axillary line. Depending on the lesion size and location of the intended thoracic cavity, the cranial and/or caudal ribs and the intercostal muscles and vessels are further cut along the axillary line, and two thoracic retractors are used to enter the thorax posteriorly at the cranial intercostal space and anteriorly at the caudal intercostal space (Figure $2 A, B$ ). With this thoracotomy technique, the whole thoracic cavity can be widely exposed and the location and length of thoracotomy along the axillary line can be adjusted depending on the lesion size. The procedure can 
be applied to an extrapleural pneumonectomy for malignant mesothelioma (8), thoracoabdominal vascular surgeries (9), or resections of any type of large thoracic tumors (10). However, care is needed for the stabilization of fragile ribs at closure and management of postoperative analgesia. Although even expert thoracic surgeons may be unfamiliar this procedure, it is a possible choice for approaching any type of large thoracic lesion and can help achieve various extended surgeries. For the present case, although the lesions were not so large, resection and cauterization of the many disseminated nodules of various sizes and locations could be achieved using this approach. Alternatively, on the basis of the disease condition, various modifications for resecting or debulking disseminated nodules can be made, such as using only a thoracoscopic approach and $\mathrm{ABC}$ to cauterize the lesions instead of using the open thoracotomy technique.

In this case, the small nodules were cauterized with $\mathrm{ABC}$ to eliminate the disseminated lesions to the extent possible. $\mathrm{ABC}$ is a noncontact, high-frequency coagulation method. Only a shallow layer of several millimeters in depth is coagulated, which prevents injuries of deeper tissues and provides better healing of the cauterized surface. Despite the cauterization of the lung surface, no air leakage occurred. In our patient, postoperative re-recurrence was found in the most caudal part of the diaphragm or upper part of the mediastinum, where application of $A B C$ was difficult, which suggests a certain effect of $A B C$. The application of $A B C$ to dissemination of thymoma has been reported (13).

For low-grade tumors for which no other effective treatment available, salvage cytoreductive surgery can be chosen for disease control, even with dissemination. For thymomas, which grows relatively slowly and are familiar to thoracic surgeons, resection of the disseminated nodules may practically be performed when the lesions are localized. A similar concept may be applied to the treatment for other intrathoracic slow-progressive disseminated tumors. Considering our patient's postoperative course, we judged that surgery would be clinically meaningful; multiple large tumors were removed, and despite the persistent disease, the patient is in good condition to date. However, the efficacy of surgery is difficult to verify, and further accumulation of clinical cases and improvement of the overall understanding of the disease to further treat LGESS are essential. As surgical therapy is extremely rarely selected for pleural dissemination of soft tissue sarcoma, its application should be thoroughly discussed in each case in a multidisciplinary conference. Our experience also suggests that rib-cross thoracotomy can be applied to disease conditions similar to our case, in addition to large thoracic tumors. We hope that this brief report and the short video will prompt further discussion on the surgical treatment that includes an unusual type of thoracotomy technique and $\mathrm{ABC}$ procedure to achieve grossly complete resection of pleural disseminations of soft tissue malignant tumors.

\section{Acknowledgments}

Funding: None.

\section{Footnote}

Reporting Checklist: The authors have completed the CARE reporting checklist. Available at https://asj.amegroups.com/ article/view/10.21037/asj-21-12/rc

Conflicts of Interest: All authors have completed the ICMJE uniform disclosure form (available at https:// asj.amegroups.com/article/view/10.21037/asj-21-12/coif). NS serves as an unpaid editorial board member of $A M E$ Surgical Fournal from December 2020 to November 2022. The other authors have no conflicts of interest to declare.

Ethical Statement: The authors are accountable for all aspects of the work in ensuring that questions related to the accuracy or integrity of any part of the work are appropriately investigated and resolved. All procedures performed in this study were in accordance with the ethical standards of the institutional and national research committees and with the Helsinki Declaration (as revised in 2013). Written informed consent was obtained from the patient for publication of this manuscript and any accompanying images. A copy of the written consent is available for review by the editorial office of this journal.

Open Access Statement: This is an Open Access article distributed in accordance with the Creative Commons Attribution-NonCommercial-NoDerivs 4.0 International License (CC BY-NC-ND 4.0), which permits the noncommercial replication and distribution of the article with the strict proviso that no changes or edits are made and the original work is properly cited (including links to both the formal publication through the relevant DOI and the license). See: https://creativecommons.org/licenses/by-nc-nd/4.0/. 


\section{References}

1. Thiel FC, Halmen S. Low-Grade Endometrial Stromal Sarcoma - a Review. Oncol Res Treat 2018;41:687-92.

2. Seagle BL, Shilpi A, Buchanan S, et al. Low-grade and high-grade endometrial stromal sarcoma: A National Cancer Database study. Gynecol Oncol 2017;146:254-62.

3. Pink D, Lindner T, Mrozek A, et al. Harm or benefit of hormonal treatment in metastatic low-grade endometrial stromal sarcoma: single center experience with 10 cases and review of the literature. Gynecol Oncol 2006;101:464-9.

4. Nakayama K, Ishikawa M, Nagai Y, et al. Prolonged long-term survival of low-grade endometrial stromal sarcoma patients with lung metastasis following treatment with medroxyprogesterone acetate. Int J Clin Oncol 2010;15:179-83.

5. Krauss K, Bachmann C, Hartmann JT, et al. Management of late recurrence of a low-grade endometrial stromal sarcoma (LGESS): treatment with letrozole. Anticancer Res 2007;27:3477-80.

6. Mizuno M, Yatabe $\mathrm{Y}$, Nawa A, et al. Long-term medroxyprogesterone acetate therapy for low-

doi: 10.21037/asj-21-12

Cite this article as: Sakakura N, Mori M, Tsukushi S, Nakada T, Takahashi Y, Kuroda H. Salvage cytoreductive surgery for pleural dissemination of low-grade endometrial stromal sarcoma using rib-cross thoracotomy with argon beam coagulation: strategies to achieve a grossly complete resectiona case report. AME Surg J 202 1;1:8. grade endometrial stromal sarcoma. Int J Clin Oncol 2012;17:348-54.

7. Amant F, Coosemans A, Debiec-Rychter M, et al. Clinical management of uterine sarcomas. Lancet Oncol 2009;10:1188-98.

8. Tsubota N. Rib cross thoracotomy for a huge thoracic lesion. Kyobu Geka 1996;49:364-6.

9. Tsukube T, Yoshimura M, Matsuda H, et al. Rib-cross thoracotomy for replacement of the thoracoabdominal or total descending aorta. J Vasc Surg 2003;37:219-21.

10. Sakakura N, Uchida T, Kitamura Y, et al. Pulmonary carcinosarcoma successfully resected using the rib-cross thoracotomy approach: report of a case. Surg Today 2014;44:175-9.

11. Tsuyoshi H, Yoshida Y. Molecular biomarkers for uterine leiomyosarcoma and endometrial stromal sarcoma. Cancer Sci 2018;109:1743-52.

12. Kakuno Y, Yamada T, Mori H, et al. Establishment and characterization of a cell line (OMC-9) originating from a human endometrial stromal sarcoma. Hum Cell 2008;21:46-55.

13. Tseng YC, Tseng YH, Tang EK, et al. A new application for argon beam coagulation: the thymoma patient with pleural recurrence. J Thorac Dis 2018;10:E355-8. 